Research Article

\title{
Study on Nonuniform Evolution Characteristics of Rock Friction and Sliding
}

\author{
Yimin Song $\mathbb{D}^{1},{ }^{1}$ He Ren $\mathbb{D}^{1},{ }^{1}$ Xiangfeng $\mathrm{Lv}\left(\mathbb{D},{ }^{2}\right.$ Tongzhen Xing $\mathbb{D}^{3},{ }^{3}$ and Yue Zhang $\mathbb{D}^{4}$ \\ ${ }^{1}$ School of Civil Engineering, North China University of Technology, Beijing 100144, China \\ ${ }^{2}$ School of Civil and Resource Engineering, University of Science and Technology, Beijing 100083, China \\ ${ }^{3}$ School of Aerospace Engineering, Beijing Institute of Technology, Beijing 100081, China \\ ${ }^{4}$ School of Civil Engineering, Beijing University of Technology, Beijing 100124, China \\ Correspondence should be addressed to Yimin Song; ssyymmok@sina.com
}

Received 19 March 2021; Revised 8 May 2021; Accepted 31 May 2021; Published 11 June 2021

Academic Editor: Mostafa S. Shadloo

Copyright $(92021$ Yimin Song et al. This is an open access article distributed under the Creative Commons Attribution License, which permits unrestricted use, distribution, and reproduction in any medium, provided the original work is properly cited.

\begin{abstract}
By means of experimental research and theoretical analysis, the nonuniform evolution characteristics of rock friction and sliding were studied. Using digital speckle correlation (DIC) as observation method, the whole process of friction and sliding of a granite specimen in double-sided shear experiment is studied. A spring slider model considering the microscopic characteristics of interface asperities was established to simulate the microscopic process of rock friction and sliding. By comparing the theoretical analysis results with the experimental results, the effect of interface nonuniformity on rock friction sliding instability is studied. The results demonstrated that, with the increase of nonuniformity of sliding interface, the degree of local instability before stickslip decreases, the stick-slip period shortens, and the value of shear drop during stick-slip period decreases. The nonuniformity of sliding interface will increase after local instability.
\end{abstract}

\section{Introduction}

With the development of geotechnical engineering projects in deep underground areas, safety accidents such as landslides and rock burst have occurred more frequently [1-4]. It is found that the rock friction and sliding process is one of the main factors causing these accidents $[5,6]$. Therefore, it is of great theoretical and practical value to deeply study the characteristics of rock friction and sliding for the prediction, early warning, and prevention of underground engineering disasters.

The research on the mechanism of rock friction sliding instability has always been one of the hot topics in the field of earthquake and geotechnical engineering. Scholars have carried out a lot of research work in this field and made many research achievements. Brace [7] and Byerlee [8] took the stick-slip phenomenon observed in the experiment and elastic rebound theory as the possible mechanism to explain the earthquake and the stages of preslip, slip nucleation, and fault healing during the stick-slip period. Dieterich [9] put forward the velocity-state friction constitutive law describing rock friction sliding. Perrin et al. [10] proposed the friction constitutive law of PRZ according to experimental research. Pan et al. [11] established the stability criterion of rock friction sliding disturbance response. Yan [12] et al. established a mechanical model of media stick-slip. He [13] obtained the sliding mode of slider system under the condition of strong and weak coupling. Song et al. [14] conducted an experimental study on the temporal and spatial evolution characteristics of deformation field and displacement during fault stick-slip process and obtained the temporal and spatial characteristics of displacement evolution during inter-stick-slip period. Miao et al. [15] studied the friction velocity dependence of rock friction and sliding through biaxial friction experiments and obtained the deformation mechanism of rock specimens with different water contents in the velocity-dependent transformation domain. Guo et al. [16] carried out rock friction and sliding experiments by different experimental methods using high-frequency experimental data observation system and obtained the relationship between stick-slip type, stress drop, and magnitude in the process of rock friction and instability sliding. Song et al. [17] used digital speckle correlation method 
to carry out experimental research on stick-slip process and analyzed the correlation between stick-slip occurrence and normal stress value. Jia et al. [18] proposed a nonlinear elasticviscoplastic damage model based on the modified Mohr-Coulomb criterion. Ma et al. [19] observed the temperature evolution of the whole process of rock friction and sliding experiment by infrared thermal imager, analyzed the temporal and spatial evolution characteristics of rock friction and sliding temperature field, and obtained the precursor characteristics of rock friction and sliding instability. Wang et al. [20] analyzed the evolution characteristics of dynamic friction coefficient, damping coefficient, and other parameters with stress and summarized the inducement of rock burst on discontinuities of rock mass. Yang et al. [21] studied the evolution characteristics of rock interface slip displacement field under cyclic loading and unloading. Lin [22, 23] studied the creep property of rocks by establishing corrected nonlinear models. Ma [24-27] simulated the coupling process of gas fracturing and analyzed the damage evolution of drilling area under different tectonic stress conditions. Although scholars have done a lot of research on the evolution characteristics of stress and deformation in the process of rock friction and sliding, the instability mechanism of rock friction and sliding is still unclear. Therefore, the combination of theoretical analysis model and experimental results is considered to carry out further research work on rock friction sliding instability mechanism.

In this paper, using DIC as observation method, the whole process of friction and sliding of a granite specimen in double-sided shear experiment is studied. A spring slider model considering the microscopic characteristics of interface asperities was established to simulate the microscopic process of rock friction and sliding. By comparing the theoretical analysis results with the experimental results, the effect of interface nonuniformity on rock friction sliding instability is studied.

\section{Experiment}

2.1. Experimental Method and Process. To study the sliding friction characteristics of rock, a double-sided shear friction test is designed, and granite with elastic modulus of about $60 \mathrm{GPa}$ is selected as the experiment specimen. The upper and lower rock blocks are fixed with the size of $300 \mathrm{~mm}$, $50 \mathrm{~mm}, 50 \mathrm{~mm}$. In addition, there is a sliding rock block in the middle, the size of which is $300 \mathrm{~mm}, 50 \mathrm{~mm}, 100 \mathrm{~mm}$. The specimen contains upper and lower sliding interfaces with an area of $0.015 \mathrm{~m}^{2}$. In order to reduce the error, the sliding interfaces were polished with 300\# emery paper before the experiment. The experimental conditions are shown in Figure 1; the left side of the fixed rock block is restrained in horizontal direction, and a vertical load of $25 \mathrm{MPa}$ is applied and kept constant. The right side of sliding rock block is loaded by displacement loading at a loading rate of $5 \mu \mathrm{m} / \mathrm{s}$. The front and back sides of the specimens are the deformation observation surfaces, where no constraints are set.

As shown in Figure 2, the experimental system includes loading system and image acquisition system. RLJW-2000 servohydraulic testing machine is selected as the experimental loading system, and the image acquisition system is built by CCD camera and observation system. The speckle field on the surface of rock mass is taken as its natural speckle field for observation. Image acquisition rate of CCD camera is 5 frames/s, image resolution is 1600 pixel $\times 1200$ pixel, and object plane resolution is $0.20 \mathrm{~mm} /$ pixel. The displacement analysis window of DIC is set to 31 pixels $\times 31$ pixels, the analysis step is 5 pixels, and the measurement accuracy is 0.01 pixels. In order to calculate the interface displacement evolution curve, three groups of monitoring points $C_{1}-C_{3}$ are arranged near the sliding interface of the specimen surface, as shown in Figure 1. In this paper, the deformation field nonuniformity index $S_{w}$ is introduced to analyze the evolution characteristics of different spatial regions of rock friction and sliding. The white area in Figure 3 is taken as the nonuniformity index $S_{w}$ calculation area.

Before the experiment, the loading device and the image acquisition system are timed to ensure that the system time is consistent. At the same time, the image acquisition system is debugged to make the camera face the specimen surface. In the experiment process, firstly, the vertical load of the specimen reaches $25 \mathrm{MPa}$ by force loading mode, and the value of vertical load remains unchanged. Then, the sliding rock block is loaded horizontally by displacement control loading, and the image acquisition system is started to collect speckle images until the end of the experiment.

2.2. Analysis Method of Nonuniform Index. The whole speckle image of the specimen collected by CCD camera is calculated by DIC, and the horizontal strain data of each pixel of the specimen is obtained. The horizontal strain values of each analysis area in Figure 2 are brought into (1), and the $S_{w}$ values of each analysis area are obtained. Then, the $S_{w}$ evolution curves of different areas are drawn.

The expression of nonuniform index $S_{w}$ [28-30] is

$$
S_{w}=w_{s} S
$$

where $S$ is the variance of strain data in the analysis area, and the calculation formula is

$$
S=S\left(X_{k}\right)=\sqrt{\frac{1}{n-1} \sum_{k=1}^{n}\left(X_{k}-\bar{X}\right)^{2}}
$$

Among them, $X_{k}$ is the deformation value of a single pixel in the analysis area, and $\bar{X}$ is the average value of $X_{k}$ of each pixel in the analysis area. $W_{s}$ is a weight for analyzing the "spatial" characteristics of regional deformation, and its calculation method is as follows:

$$
w_{s}=S\left(X_{k}^{*}\right)=\sqrt{\frac{1}{n-1} \sum_{k=1}^{n}\left(X_{K}^{*}-\overline{X^{*}}\right)^{2}}
$$

$X^{*}$ is calculated from the data matrix $X$ of the deformation field by crossing the matrix $B$, as shown in the following formula: 


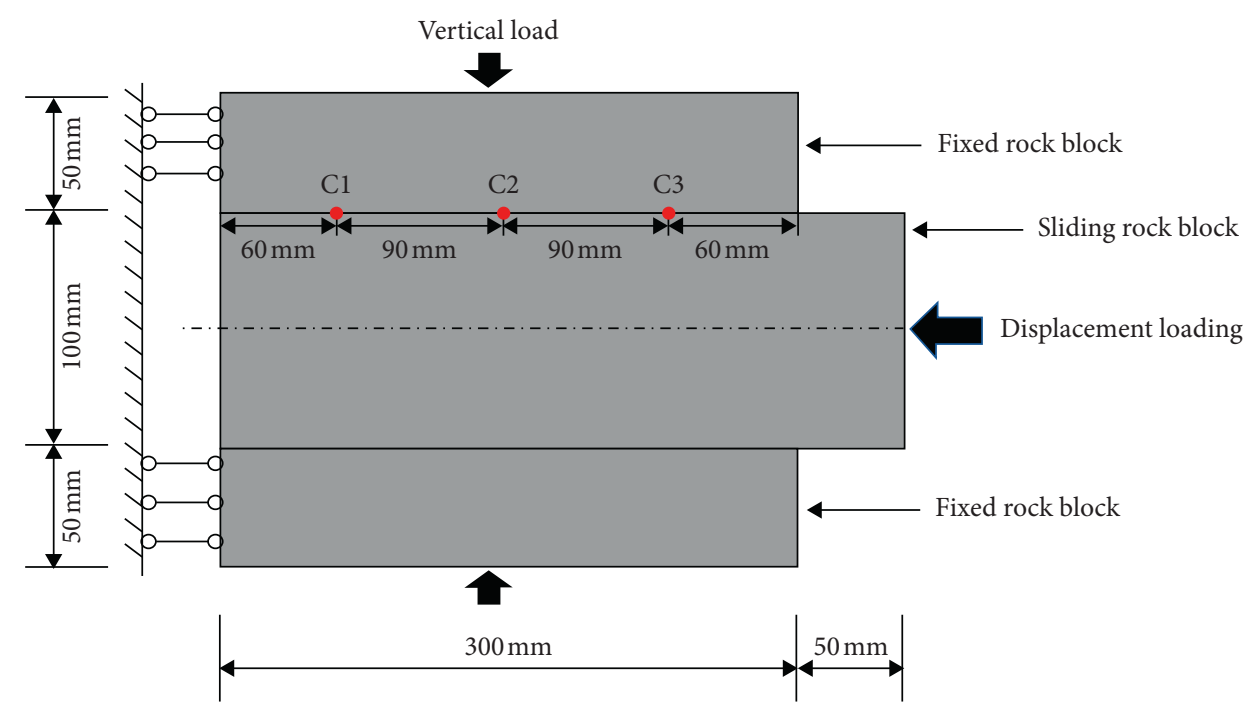

FIgURE 1: The experimental model.

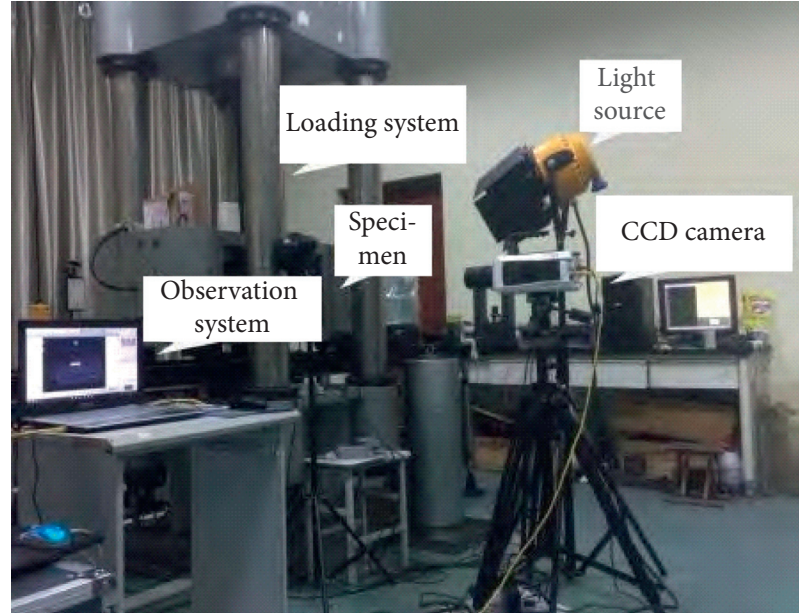

Figure 2: The picture of the experimental system.

$$
X^{*}=X \otimes B .
$$

$B$ is a square matrix whose elements are all 1 and whose size is $m$.

2.3. Analysis of Experimental Results. It can be seen from Figures 4 and 5 that the shear stress of the specimen changes from slow increase to linear increase, nonlinear increase, and periodic stick-slip stage in turn. In the stage of slow increase of shear stress, the interface sliding displacement is small, and the value of $S_{w}$ in different areas is small. In the nonlinear growth stage of shear stress, the interface slip increases nonlinearly, the $S_{w}$ curve shows a wave evolution trend, and there are obvious time differences in the wave trend of $S_{w}$ curves in different regions. In the periodic stickslip stage, the shear stress suddenly drops, the sliding displacement of the corresponding interface suddenly increases, and the value of $S_{w}$ in different areas suddenly changes. During the inter-stick-slip period, the shear stress increases approximately linearly, and the corresponding interface sliding displacement changes slightly. The $S_{w}$ curve

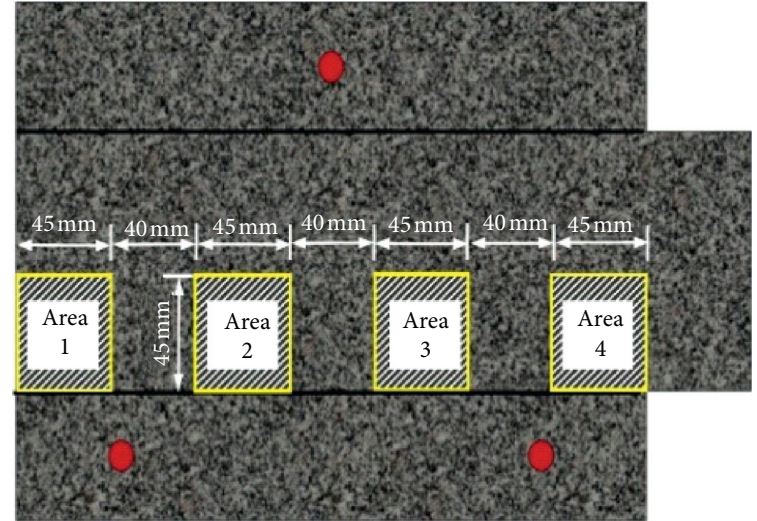

FIgURE 3: $S_{w}$ calculation area.

is characterized by wave evolution, the wave trends in different regions are obviously unsynchronized, and the values of $S_{w}$ in different region are different.

The analysis shows that the shear stress increases slowly and linearly, the sliding surface is in static friction state, and the value of nonuniformity index is small. Because of the compaction effect of normal stress on the micro-concave-convex body of sliding interface, the nonuniformity index increases approximately linearly. In the stage of nonlinear growth of shear stress, the sliding surface changes from static friction to sliding friction, which makes the region near the loading end show local sliding instability, while the nonuniformity index grows rapidly, and calculation area 4 has obvious fluctuation evolution characteristics. In the periodic stick-slip stage and inter-stick-slip stage, the sliding surface is in a viscous state, and the nonuniformity fluctuates. During the stick-slip period, the slip surface slides and the inhomogeneity drops.

It can be seen that the nonuniformity of sliding interface is closely related to the instability of rock friction and sliding. Further, a spring slider model is established to study the effect of sliding interface nonuniformity on rock friction sliding instability. 


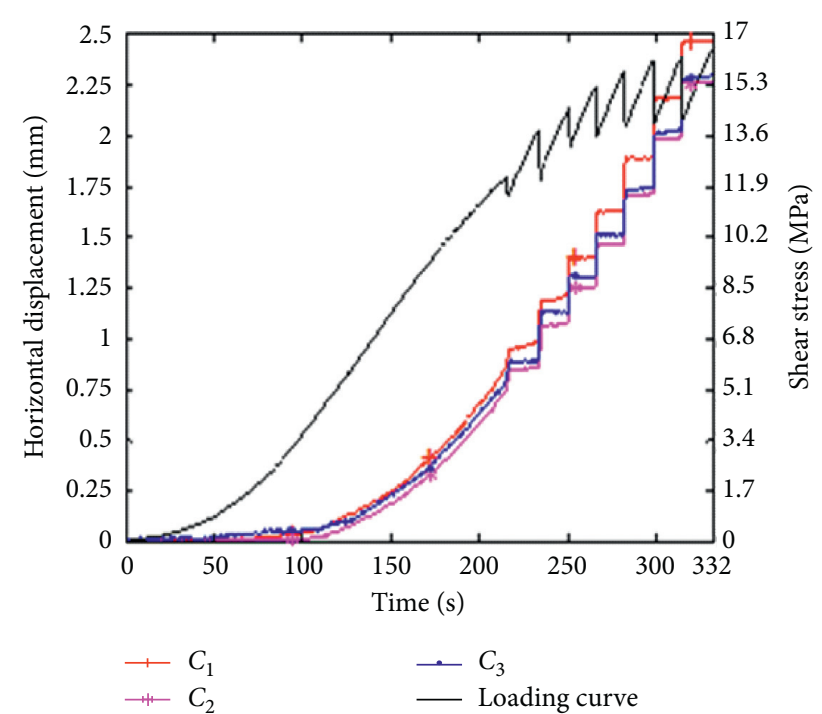

FIGURE 4: The loading curve and interface displacement evolution curve.

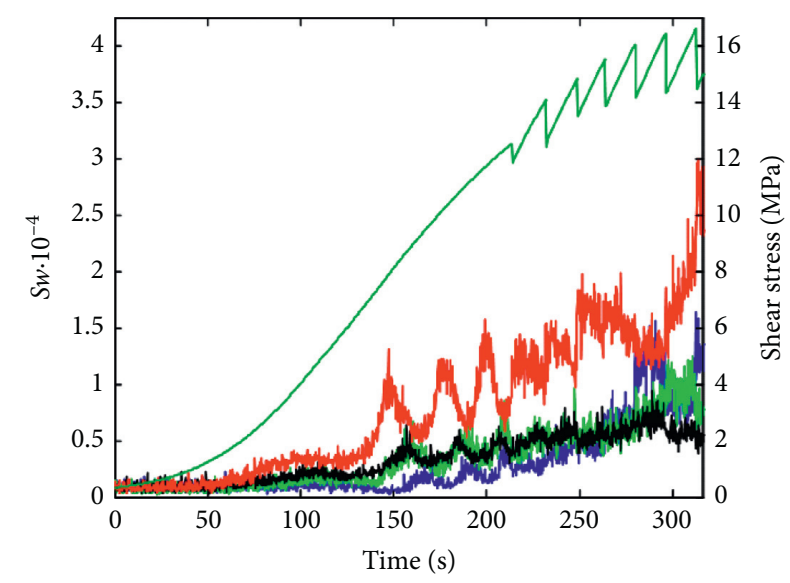

Loading rate: $5 \mu \mathrm{m} / \mathrm{s}$

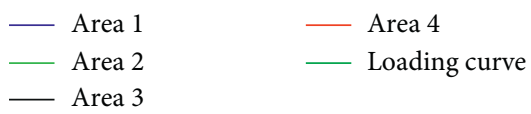

Figure 5: The loading curve and $S_{w}$ evolution curve.

\section{Numerical Simulation of Rock Frictional Sliding Instability}

3.1. Theoretical Model of Rock Friction and Sliding. Based on the finite element method, the sliding rock block in the specimen model of two-sided shear friction experiment is discretized into finite elements, as shown in Figure 6, where Part A is the sliding rock block and Part B is the fixed rock block. The spring connection slider simulates the action between sliders; that is, Part A of each element is connected by a spring. The interface spring is used to simulate the contact between Part A and Part B, and the connection state of the interface spring is used to simulate the microscopic behavior of unstable shearing and recontact of asperities in the process of rock friction and sliding.
In the model, the sliders are affected by the force between sliders, interfacial friction, damping force, and inertial force. Among them, the first slider at the loading end is subjected to the external force exerted by the loading system in addition to the above-mentioned acting force. The dynamic equation of the whole system is shown in (6), and the dynamic equation of a single slider is shown in (5).

$$
\begin{aligned}
m \ddot{\mu}_{i}+2 \eta \dot{\mu}_{i}+2 K_{b} \mu_{i} & =\eta \dot{\mu}_{i-1}+K_{b} \mu_{i-1}+K_{b} \mu_{i+1}-F_{f}, \\
M \ddot{\mu}(t)+C \dot{\mu}(t) K \mu(t) & =Q(t),
\end{aligned}
$$

where $i$ is slider number, $m$ is mass of single slider, $\eta$ is damping coefficient of single slider, $K_{b}$ is equivalent stiffness of single slider, $\mu_{i}$ is displacement of slider $i, \dot{\mu}_{i}$ is sliding speed of slider $i, \ddot{\mu}_{i}$ is sliding acceleration of slider $i, F_{f}$ is friction force of interface spring, $M$ is the system mass matrix, $K$ is the system stiffness matrix, $C$ is the system damping matrix, and $Q$ is the load vector, that is, the resultant force of the force $F_{s}$ applied by the loading system and the interfacial friction $F_{f}$.

The central difference method is used to simulate the dynamic behavior of rock friction and sliding, and the acceleration and velocity are expressed as follows:

$$
\begin{aligned}
& \ddot{\mu}_{t}=\frac{1}{\Delta t^{2}}\left(\mu_{t-\Delta t}-2 \mu_{t}+\mu_{t+\Delta t}\right), \\
& \dot{\mu}_{t}=\frac{1}{2 \Delta t}\left(-\mu_{t-\Delta t}+\mu_{t+\Delta t}\right) .
\end{aligned}
$$

By substituting (7) and (8) into (6), the recurrence formula of displacement calculation is obtained:

$$
\left(\frac{1}{\Delta t^{2}} M+\frac{1}{2 \Delta t} C\right) \mu_{t+\Delta t}=Q_{t}-\left(K-\frac{2}{\Delta t^{2}} M\right) \mu_{t}-\left(\frac{1}{\Delta t^{2}} M-\frac{1}{2 \Delta t} C\right) \mu_{t-\Delta t} .
$$
(9) is

The formula of initial velocity and initial acceleration in

$$
\begin{aligned}
\mu_{-\Delta t} & =\mu_{0}-\Delta t \dot{\mu}_{0}+\frac{\Delta t}{2} \ddot{\mu}_{0}, \\
\ddot{\mu}_{0} & =M^{-1}\left(Q_{0}-C_{0} \dot{\mu}_{0}-K \mu_{0}\right) .
\end{aligned}
$$

The friction force $f_{i}$ on the interface spring is calculated by incremental law. Set the tensile strength $f_{t}$ of the spring, and judge that the spring fails when the calculated friction force $f_{i}$ on the interface spring exceeds $f_{t}$; that is, the slider is disconnected from the spring of the sliding interface and can be connected to work again after $\tau_{t h}$, so the calculation method of friction force of a single spring is shown in

$$
f_{i}(t)= \begin{cases}f_{i}(t-\Delta t)+k_{i} \Delta x, & f_{i}(t-\Delta t)<f_{t}, \\ 0, & f_{i}(t-\Delta t)>f_{t} \| t<\tau_{t h} .\end{cases}
$$

Thereinto, $f_{i}(t)$ and $f_{i}(t-\Delta t)$ are the frictional forces of the interface spring at $t$ and $t-\Delta t$, respectively, $k_{i}$ is the stiffness value of the $i_{\text {th }}$ interface spring of the slider, and $\Delta x$ is the displacement change of the slider in $\Delta t$ time period. 


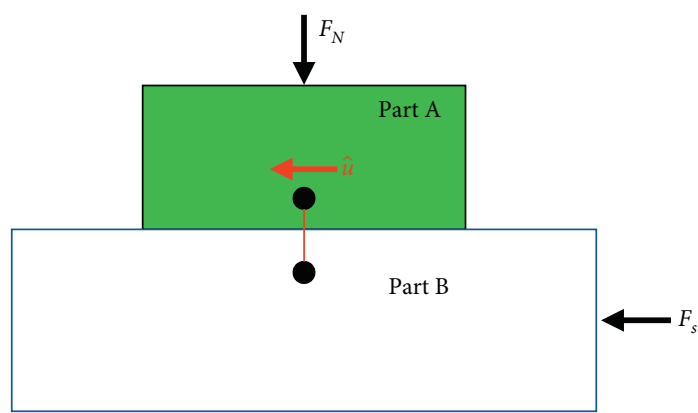

(a)

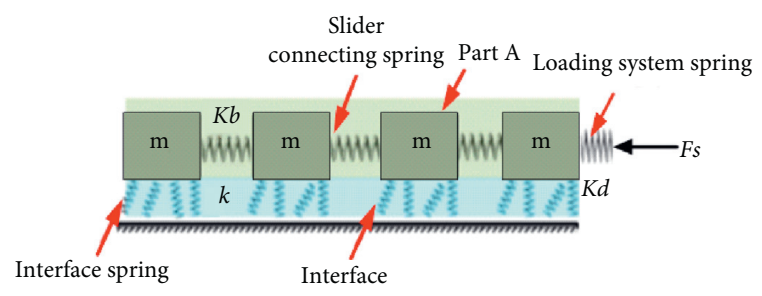

(b)

FiguRE 6: The rock friction sliding spring slider model: (a) unit body; (b) spring slider system.

By comparing the space-time evolution diagram of interface friction [29] calculated by setting different sliding numbers, it is determined that the number of sliding blocks is 70 .

The calculation method of slider stiffness $K_{b}$ is shown in

$$
K_{b}=\frac{E W H}{L} .
$$

$W$ is the thickness of slider with a value of $50 \mathrm{~mm} ; H$ is the height of slider with a value of $100 \mathrm{~mm}$; $E$ is the elastic modulus of material with a value of $60 \mathrm{GPa}$; and $L$ is the length of a single slider, and its value is $5 \mathrm{~mm}$. With (13), $K_{b}$ is $6.0 \times 10^{10} \mathrm{~N} / \mathrm{m}$.

The release time $\tau_{\text {th }}$ of the interface spring is estimated by the time when the shear wave passes through a single slider as shown in

$$
\tau_{\text {th }}=\frac{L}{v_{s}} .
$$

Among them, $v_{s}$ is the propagation velocity of rock shear wave, the value is set at $3500 \mathrm{~m} / \mathrm{s}$, and the release time of interface spring is calculated as $1.0 \times 10^{-7} \mathrm{~s}$ by introducing (13).

The shear stiffness of two-sided shear test is twice that of single-sided shear test, so the calculation method of average stiffness $\langle k\rangle$ of interface spring is shown in

$$
\langle k\rangle=\frac{2 K_{s}}{N_{s}},
$$

where $K_{s}$ is the shear stiffness of slider, and the calculation method is as follows:

$$
K_{s}=\frac{G W L}{H}
$$

In order to save the time of solving the model, the loading rate is set to $5 \mathrm{~mm} / \mathrm{s}$ and the loading step is set to $1 \times 10^{-7} \mathrm{~s}$ without affecting the numerical analysis results. The stiffness $K_{d}$ of the loading system is calculated by the loading curve of the specimen with a loading rate of $5 \mu \mathrm{m} / \mathrm{s}$, and the calculation method is as follows:

$$
K_{d}=\frac{\Delta F}{\delta} .
$$

Here, $\Delta F$ is the load force drop when the stick-slip event occurs during the experiment, $\delta$ is the interface sliding displacement value of the stick-slip event, and $K_{d}$ is $6.6 \times 10^{8} \mathrm{~N} / \mathrm{m}$.
3.2. Nonuniformity Simulation Method. In order to effectively simulate the microscopic behavior of "occlusionfailure-occlusion" of interface asperities, multiple sets of interface springs are connected to each slider in the model, and the interface springs deform with the slider to generate tension. When the tensile force reaches its tensile strength, the interface spring is released. However, after $\tau_{\text {th }}$ time, the disconnected interface spring is connected with the slider again.

In order to simulate the strength nonuniformity of interface asperities, Gaussian distribution is used to describe the strength distribution of interface springs. According to the previous calculation method [30], the average strength of interface spring is set to be $23.8 \mathrm{~N}$. Set the discrete coefficients of interface spring strength to be $0.05,0.10$, and 0.20 , and draw the Gaussian distribution diagram of interface spring strength $f_{t}$, as shown in Figure 7. Later, different spring dispersion coefficients are used to describe the different nonuniformity of the sliding interface of the specimen.

The spring stiffness of single slider interface is calculated according to the average strength $\bar{f}_{t}$ and average stiffness $\langle k\rangle$, as shown in (13). The spring stiffness distribution curve of a single slider interface is shown in Figure 8.

\section{Analysis of Numerical Simulation Results of Rock Friction Sliding Instability}

Figure 9 shows the theoretical model loading curve and interface displacement evolution curve under the interface spring strength dispersion coefficient of 0.1 . It can be seen that the load increases linearly at the initial stage and then increases nonlinearly after a period of time until stick-slip occurs. This trend is basically consistent with the evolution trend of loading curve in experimental results. In addition, it can be seen that the evolution trend and numerical value of the sliding displacement of the specimen are in good agreement with the experimental results. Therefore, it is judged that the theoretical model of rock friction and sliding can explain the experiment well, and the instability process of rock friction and sliding can be studied deeply according to the calculation results of the model.

Figures $10-12$ and Table 1 show the results of the theoretical model. Figure 11 is the sketch of a partial 


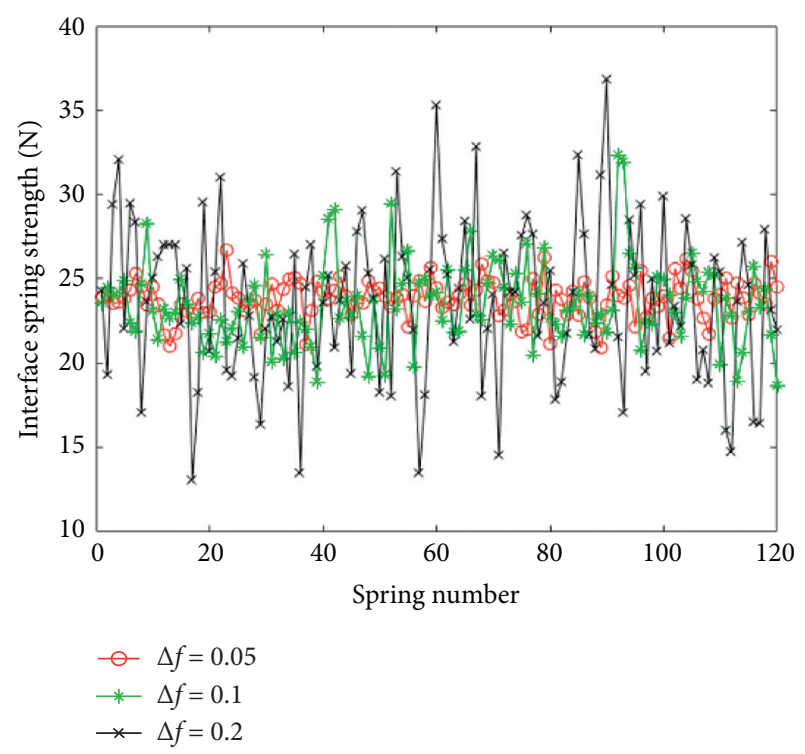

FIGURE 7: The interface spring intensity distribution diagram with standard deviation of different strength distributions.

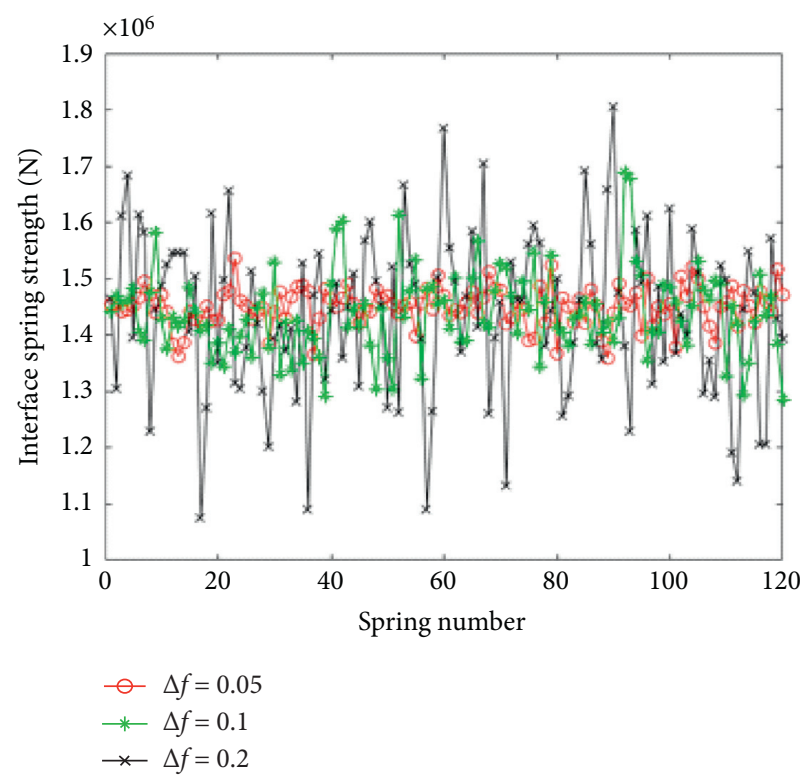

FIGURE 8: The interfacial connection spring stiffness distribution diagram with standard deviation of different strength distributions.

enlarged view selected from the nonlinear stage of the loading curve. In the nonlinear stage, the loading curves are obviously different with different uniformity. With the increase of nonuniformity, the curve is smoother. According to the analysis of the friction evolution curve of a single slider in Figure 12, it can be seen that before stickslip, that is, the nonlinear stage of macroscopic loading force, the slider will experience many sudden drops of friction force. Moreover, with the increase of nonuniformity, the sudden drop decreases obviously. However, the sudden drop of friction force is due to the failure of tensile strength of a large number of interface springs, which leads to the instability of slider. It can be seen that,

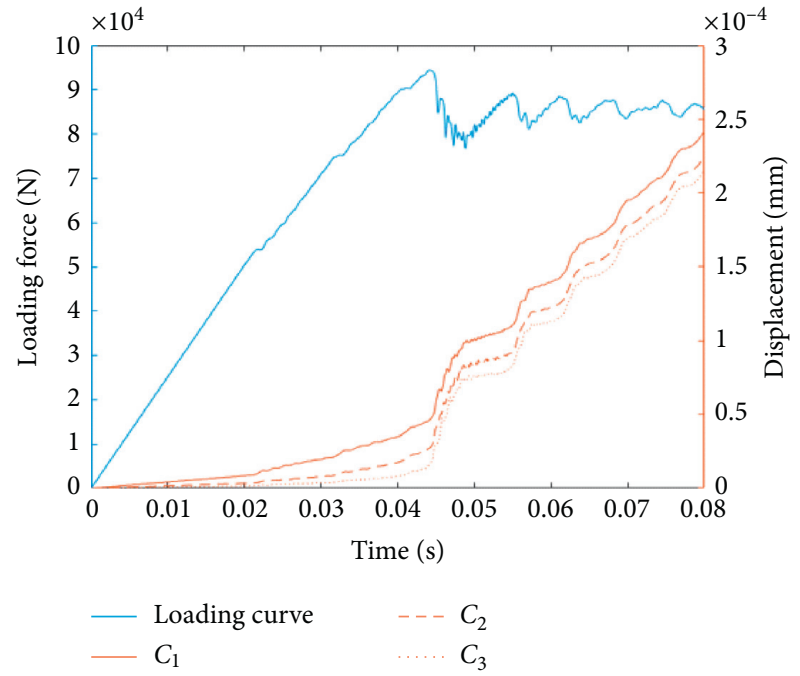

Figure 9: The numerical model loading curve and sliding displacement evolution curve.

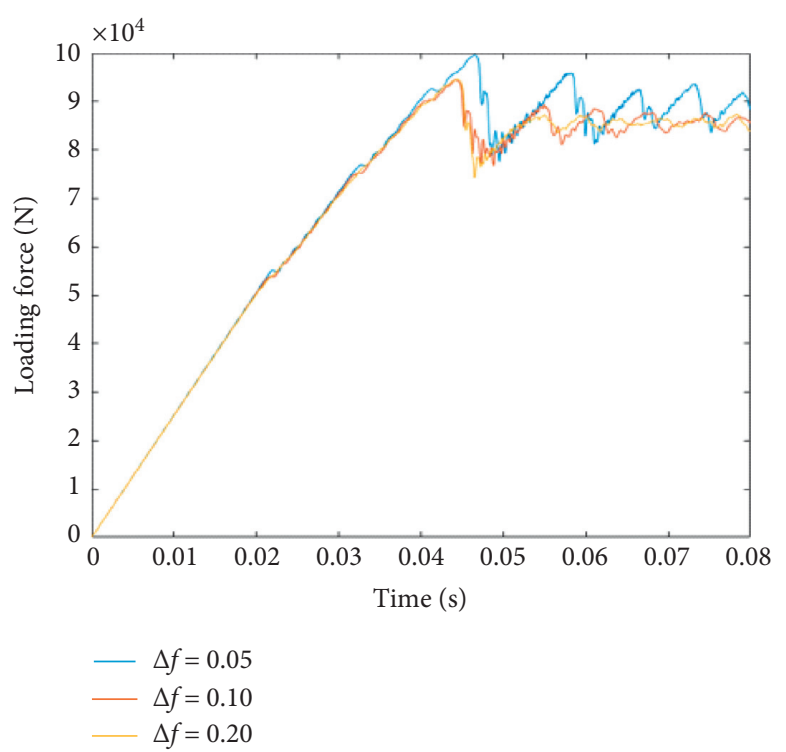

Figure 10: Loading curves of numerical model under different uniformity conditions.

with the increase of the nonuniformity of the sliding interface, the local instability degree of the sliding interface before sticking and sliding decreases. By comparing the first instability under different uniformity with multiple instability events under the same uniformity, it is found that the evolution trends of the two are similar. This phenomenon also exists in the $S_{w}$ evolution curve in the experimental results, and the $S_{w}$ value after local instability is higher than that before instability. It is considered that the interface inhomogeneity changes and increases after every instability event.

Take curves in Figure 10 as the research object to analyze the difference of stick-slip mechanism under different uniformity conditions. On the time scale of $80 \mathrm{~ms}$, when $\Delta f=0.05$ and 0.1 , the specimen experienced four 


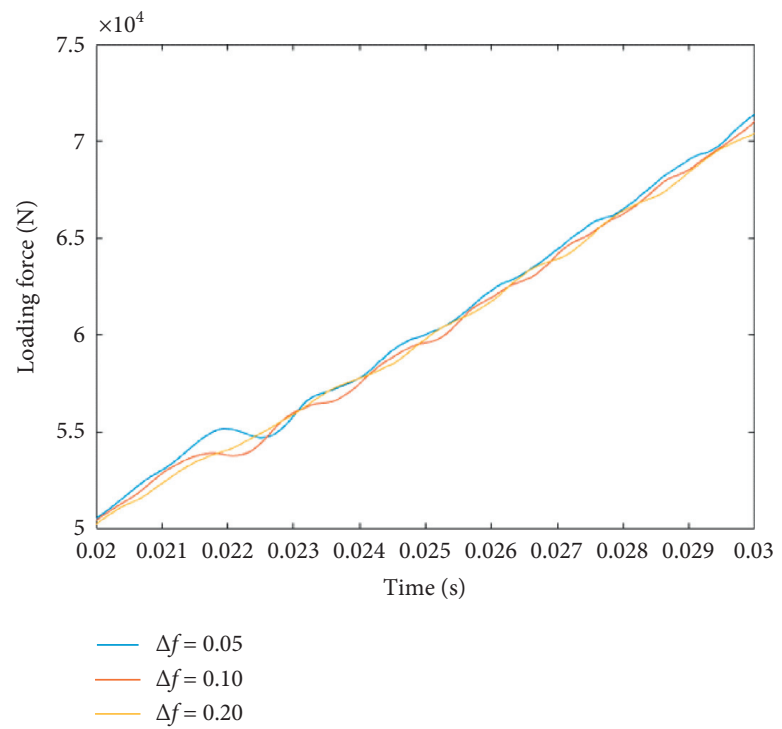

Figure 11: The partial enlarged detail of nonlinear stage.

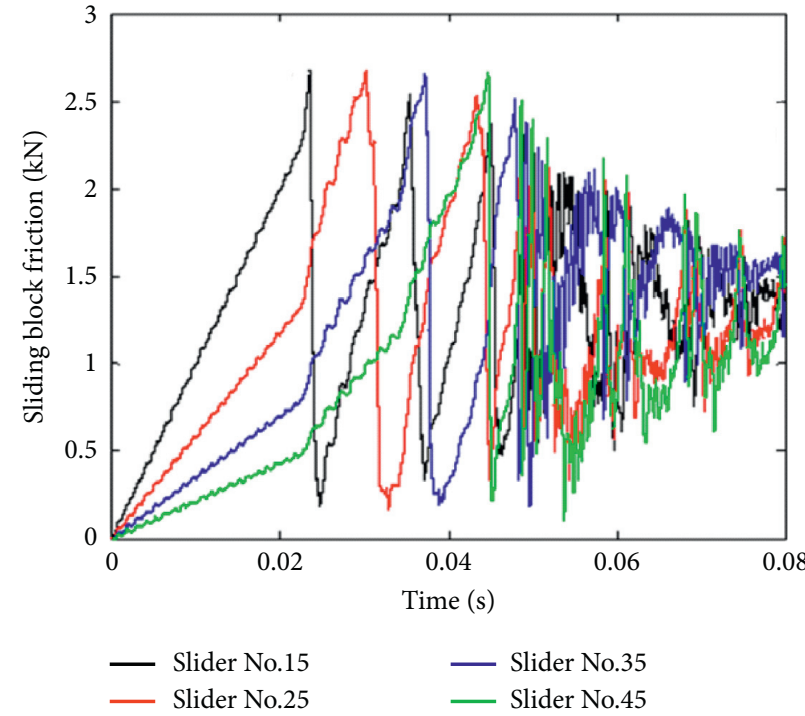

(a)

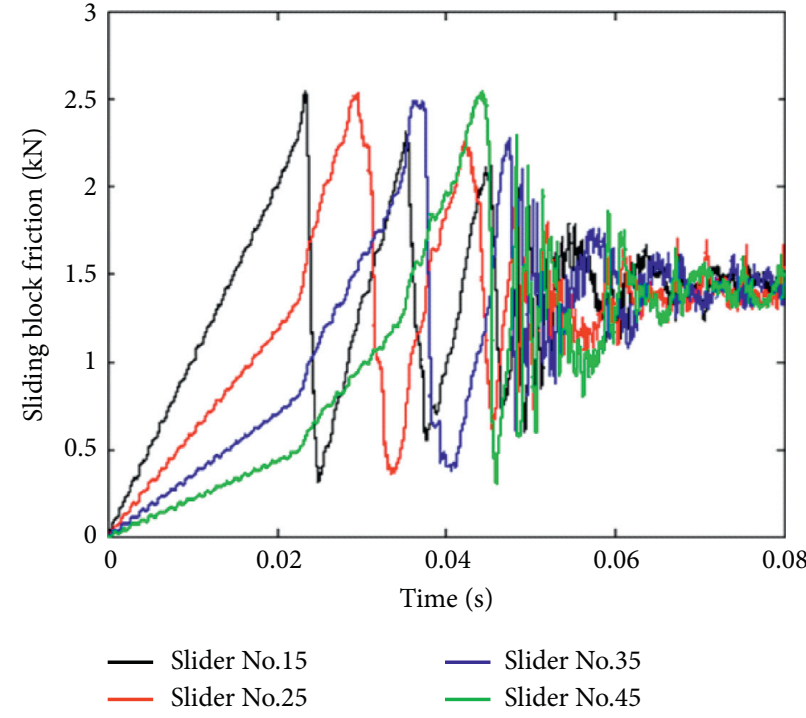

(b)

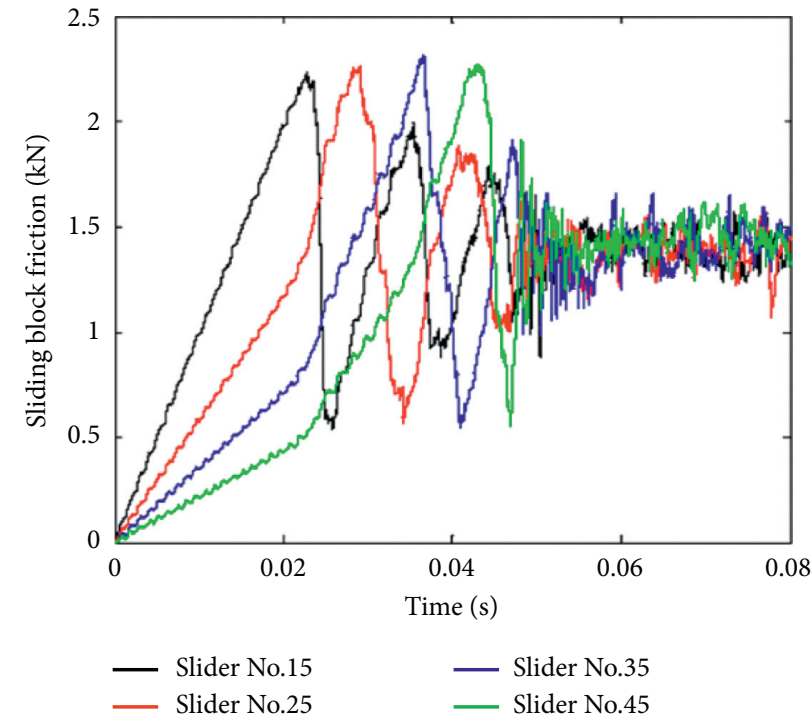

(c)

FIgURE 12: The slider friction evolution curve: (a) $\Delta f=0.05$; (b) $\Delta f=0.10$; (c) $\Delta f=0.20$. 
TABLe 1: Viscous slip event shear derating table $(\mathrm{kN})$.

\begin{tabular}{lcccccc}
\hline Stick-slip times & 1 & 2 & 3 & 4 & 5 & 6 \\
\hline$\Delta f=0.05$ & 18.40 & 8.89 & 13.15 & 5.63 & & \\
$\Delta f=0.10$ & 15.92 & 11.15 & 6.72 & 4.04 & & \\
$\Delta f=0.20$ & 14.97 & 6.43 & 3.09 & 2.25 & 2.24 & 2.32 \\
\hline
\end{tabular}

stick-slip processes; when $\Delta f=0.20$, the specimen experienced six stick-slip processes. It can be seen from Table 1 that, with the increase of interface nonuniformity, the shear drop value of the specimen decreases during stickslip period. In other words, with the improvement of interface inhomogeneity, the stick-slip period of specimen is shortened, and the value of shear drop in stick-slip period is reduced. A similar rule can be seen by taking a single slider as the research object in Figure 12. When $\Delta f$ increases, the fluctuation range of slider friction in stick-slip period is obviously reduced, and the fluctuation period is shortened. From the model point of view, when the strength dispersion coefficient of interface spring increases, there will be more springs with smaller strength, and the number of interface springs participating in the sudden decrease of friction force will decrease. Before the slide block is sheared, the springs with smaller strength are destroyed first, and when a large number of springs are destroyed, these early-destroyed springs are connected again. Therefore, the fluctuation interval of friction force of a single slider will be reduced, and the fluctuation process will be shortened. The fluctuation range and fluctuation period of the whole interface friction force will also decrease. It is known that there is a good corresponding relationship between shear force and interfacial friction force in the whole process, so the decrease of shear force dropping value and the shortening of stick-slip period can be better explained.

\section{Conclusion}

(1) By comparing the theoretical analysis results with the experimental results of two-sided shear friction, it is considered that the instability process of rock friction and sliding can be deeply studied according to the theoretical model of rock friction and sliding.

(2) By analyzing the loading curve and sliding block friction evolution curve in the nonlinear stage, it is concluded that the specimen will have local instability many times before stick-slip occurs. The degree of local instability will decrease with the increase of interface inhomogeneity. After the specimen undergoes local instability, the interface inhomogeneity will increase.

(3) By observing the loading curve of periodic stick-slip stage, the friction evolution curve of slider, and the shear drop value table of stick-slip event under different uniformity conditions, it is concluded that, with the improvement of the nonuniformity of sliding interface, the stick-slip period of specimen is shortened, and the shear drop value in stick-slip stage is reduced.

\section{Data Availability}

The data used to support the findings of this study are available from the corresponding author upon request.

\section{Conflicts of Interest}

The authors declare no conflicts of interest.

\section{Acknowledgments}

This work was supported by the National Natural Science Foundation of China (nos. 51474013 and 51774015).

\section{References}

[1] Q. X. Qi, Z. Z. Gao, S. Wang et al., "The theory of rockburst led by structure failure of bedded coal-rock mass," Coal Mining Technology, vol. 30, no. 2, pp. 14-17, 1998.

[2] L. Y. PanH. Z. Yang et al., "Dilatancy theory for identification of premonitory information of rock burst," Chinese Journal of Rock Mechanics and Engineering, vol. 23, no. z1, pp. 45284530, 2004.

[3] G. M. Yu and H. P. Xie, "Numerical simulation of fractal effect induced by activation of fault after coal extraction," Journal of China Coal Society, vol. 23, no. 4, pp. 396-400, 1998.

[4] X. X. Miao, X. M. Cui, J. A. Wang et al., "The height of fractured water-conducting zone in undermined rock strata," Engineering Geology, vol. 120, no. 1, 2011.

[5] W. M. Han, T. H. Kang, J. J. Li et al., "Influence of surface topography on constitutive parameter of rock frictional sliding," Chinese Journal of Rock Mechanics and Engineering, vol. 32, no. 8, pp. 1632-1639, 2013.

[6] S. J. Liu, L. X. Wu, J. Z. Wang et al., "Remote sensing-rock mechanics(VI) - features of rock friction-sliding and analysis on its influence factors," Chinese Journal of Rock Mechanics and Engineering, vol. 23, no. 8, pp. 1247-1251, 2004.

[7] W. F. Brace and J. D. Byerlee, "Stick-slip as a mechanism for earthquakes,” Science, vol. 153, no. 3739, pp. 990-992, 1966.

[8] J. D. Byerlee, "The mechanics of stick-slip," Tectonophysics, vol. 9, no. 5, pp. 475-486, 1970.

[9] J. H. Dieterich, "Earthquake nucleation on faults with rate and state-dependent strength," Tectonophysics, vol. 211, no. 1-4, pp. 115-134, 1992.

[10] G. Perrin, J. R. Rice, and G. Zheng, "Self-healing slip pulse on a frictional surface," Journal of the Mechanics and Physics of Solids, vol. 43, no. 9, pp. 1461-1495, 1995.

[11] Y. S. Pan, L. G. Wang, M. T. Zhang et al., "The theoretical and testing study of fault rock burst," Chinese Journal of Rock Mechanics and Engineering, vol. 17, no. 06, pp. 642-649, 1998.

[12] Y. G. Yan, G. R. Feng, Y. D. Zhai et al., "Stick-slip conditions and dynamics analysis of coal rock burst," Journal of China Coal Society, vol. 35, no. S1, pp. 19-21, 2010.

[13] C. R. He, "Interaction of double slider system under velocity and state dependent friction constitutive relationship," Science in China, vol. 33, no. S1, pp. 53-59, 2003.

[14] Y. M. Song, S. P. Ma, X. B. Yang et al., "Experimental study on the dynamic displacement evolution of fault in stick-slip process," Chinese Journal of Geophysics, vol. 55, no. 1, pp. 171-179, 2012.

[15] L. Miao, S. L. Ma, L. F. Hou et al., "Velocity-dependence transition of friction for halite gouge and its significance implication in seismology," Chinese Journal of Geophysics, vol. 55, no. 10, pp. 3307-3317, 2012. 
[16] L. L. Guo, L. Q. Liu, J. Ma et al., "The magnitude estimation in stick-slip experiments and analysis of stress drop," Chinese Journal of Geophysics, vol. 57, no. 03, pp. 867-876, 2014.

[17] Y. M. Song, S. P. Ma, X. B. Yang et al., "Experimental investigation on instability transient process of fault rockburst," Chinese Journal of Rock Mechanics and Engineering, vol. 30, no. 4, pp. 812-817, 2011.

[18] S. P. Jia, L. W. Zhang, B. S. Wu, H. D. Yu, and J. X. Shu, "A coupled hydro-mechanical creep damage model for clayey rock and its application to nuclear waste repository," Tunnelling and Underground Space Technology, vol. 74, Article ID 230246, 2018.

[19] J. Ma, L. Q. Liu, P. X. Liu et al., "Thermal precursory pattern of fault unstable sliding: an experimental study of en echelon faults," Chinese Journal of Geophysics, vol. 50, no. 4, pp. 1141-1149, 2007.

[20] L. G. Wang, M. T. Meng, Y. J. Wang et al., "The imitating test study of sliding process for the single fault rock sample," Rock and Soil Mechanics, vol. 18, no. 6, pp. 82-89, 2017.

[21] X. B. Yang, J. Zhou, Y. M. Song et al., "Evolution characteristics of sliding displacement of rock interface under cyclic loading," Journal of China Coal Society, vol. 44, no. 10, pp. 3041-3048, 2019.

[22] H. Lin, X. Zhang, Y. Wang et al., "Improved nonlinear Nishihara shear creep model with variable parameters for rock-like materials," Advances in Civil Engineering, vol. 2020, no. 3, Article ID 7302141, 15 pages, 2020.

[23] H. Lin, X. Zhang, R. Cao et al., "Improved nonlinear burgers shear creep model based on the time-dependent shear strength for rock," Environmental Earth Sciences, vol. 79, no. 6, 2020.

[24] D. Ma, J. Zhang, H. Duan et al., "Reutilization of gangue wastes in underground backfilling mining: overburden aquifer protection," Chemosphere, vol. 264, no. Pt 1, Article ID 128400, 2021.

[25] D. Ma, H. Duan, X. Li, Z. Li, Z. Zhou, and T. Li, "Effects of seepage-induced erosion on nonlinear hydraulic properties of broken red sandstones," Tunnelling and Underground Space Technology, vol. 91, no. SEP., p. 102993, 2019.

[26] D. Ma, H. Duan, J. Liu, X. Li, and Z. Zhou, "The role of gangue on the mitigation of mining-induced hazards and environmental pollution: an experimental investigation," Science of the Total Environment, vol. 664, no. MAY 10, pp. 436-448, 2019.

[27] D. Ma, H. Duan, Q. Zhang et al., "A numerical gas fracturing model of coupled thermal, flowing and mechanical effects," Computers, Materials and Continua, vol. 65, no. 3, pp. 2123-2141, 2020.

[28] Y. M. Song, Y. Zhang, H. L. Xu et al., "Study on creep-slip and stick-slip deformation evolution of rock based on non-uniform characteristics," Rock and Soil Mechanics, vol. 41, no. 2, pp. 363-371, 2020.

[29] L. M. Dou and X. Q. He, "Model for rork burst failure and its critical values of acoustic and electromagnetic emission," Journal of China University of Mining and Technology, vol. 33, no. 5, pp. 14-18, 2004.

[30] Y. Y. Cao, The Experimental Study of Rock Stick-Slip Dynamic Process, Beijing Institute of Technology, Beijing, China, 2015. 